\title{
Brain imaging in a patient with hemimicropsia
}

\author{
J. Kassubek*, M. Otte, T. Wolter, M.W. Greenlee, T. Mergner, C.H. Lücking \\ Neurologische Universitätsklinik, Universität Freiburg, Freiburg, Germany
}

Received 8 June 1998; accepted 2 March 1999

\begin{abstract}
Hemimicropsia is an isolated misperception of the size of objects in one hemifield (objects appear smaller) which is, as a phenomenon of central origin, very infrequently reported in literature. We present a case of hemimicropsia as a selective deficit of size and distance perception in the left hemifield without hemianopsia caused by a cavernous angioma with hemorrhage in the right occipitotemporal area. The symptom occurred only intermittently and was considered the consequence of a local irritation by the hemorrhage. Imaging data including a volume-rendering MR data set of the patient's brain were transformed to the 3-D stereotactic grid system by Talairach and warped to a novel digital 3-D brain atlas. Imaging analysis included functional MRI (fMRI) to analyse the patient's visual cortex areas (mainly V5) in relation to the localization of the hemangioma to establish physiological landmarks with respect to visual stimulation.

The lesion was localized in the peripheral visual association cortex, Brodmann area (BA) 19, adjacent to BA 37, both of which are part of the occipitotemporal visual pathway. Additional psychophysical measurements revealed an elevated threshold for perceiving coherent motion. which we relate to a partial loss of function in V5, a region adjacent to the cavernoma.

In our study, we localized for the first time a cerebral lesion causing micropsia by digital mapping in Talairach space using a 3-D brain atlas and topologically related it to fMRI data for visual motion. The localization of the brain lesion affecting BA 19 and the occipitotemporal visual pathway is discussed with respect to experimental and case report findings about the neural basis of object size perception. (C) 1999 Elsevier Science Ltd. All rights reserved.
\end{abstract}

Keywords: Micropsia; Size constancy; Cavernoma; Brain atlas; Functional MRI

\section{Introduction}

Hemianopic defects in vision are frequent phenomena in the daily neuro-ophthalmological clinical routine. Less common are complaints about more complex changes in visual perception following lesions of the cerebral cortex. These deficits are of interest since they can shed light on the functional role of the lesioned cortical area. Examples are dysmetropsia (also called dysmegalopsia) and metamorphopsia, which reflect disorders of visual processing causing subjective

\footnotetext{
* Corresponding author. Neurologische Klinik und Poliklinik der Universität Freiburg, Breisacher Str. 64 79106, Freiburg, Germany. Tel.: +49-761-2705001 (work); fax: +49-0761-5559588.

E-mail address: kassubek@nz11.ukl.uni-freiburg.de (J. Kassubek)
}

distortions in the size or the shape of the perceived object, respectively. In dysmetropsia, objects may appear either reduced (micropsia) or enlarged (macropsia) relative to their normal size. Micropsia is more frequently reported than macropsia, but both are rather infrequent phenomena, even more so if occurring as isolated symptoms. More common is the combination of these perceptual abnormalities with visual field loss following extensive central lesions [11]).

The phenomenon of metamorphopsia appears to be related to the perceptual mechanism of size constancy (phenomenologically, an object appears to retain its size despite substantial variations in viewing distance). Normally, the perceived size of an image is scaled according to its perceived distance, depending on a number of depth and distance cues. Errors of scaling 
may occur with abnormalities in retinal image size, disturbances related to the binocular disparity and the central interpretation of the received afferent inputs.

In cases where dysmetropsia occurs monocularly, it is likely to be due to retinal diseases which cause displacement of the receptor cells, for instance excessive separation of the photoreceptors by edematous fluid (like in central serous retinopathy; see [12]). Furthermore, dysmetropsia can result from a size reduction of the retinal image due to an accommodation increase of the lens influenced by the distances between the object plane and the pupil and the accommodation level, which is a phenomenon called accommodative micropsia [24]. A similar mechanism may underly the cases in which dysmetropsia occurs associated with muscular diseases such as ocular myasthenia [14]. In cases of central origin, micropsia is mostly described as a transient phenomenon, occurring as prodroma of, or simultaneously with, temporal lobe epileptic seizures or migraine [21]. It is then either accompanied by various symptoms of temporal lobe epilepsy, or it presents-very rarely-as a form of misperception amongst others (metamorphopsia, dyschromatopsia) in purely visual seizures. Cases are reported in which micropsia was classified as a psychopathological phenomenon without evidence of neurological dysfunction ('psychogenic micropsia') [20], but these cases may possibly reflect undetected visual seizures.

There are only few reports in which micropsia (or hemimicropsia, i.e., apparent reduction of the size of objects when presented in one hemifield) have been brought in association with a focal cerebral lesion, for instance a brain tumor, hemorrhage or ischaemic infarction. However, the lesions reported are rather scattered over extended regions of the brain, such as the occipital lobe (micropsia associated with a homonymous loss of the visual field) [12], the retrosplenial area [6], the chiasm [1] and visual association areas [3]. Wieser found micropsia/macropsia mainly when stimulating neocortical regions in the temporal-occipital border zone [32]. A clearcut localization of a cortical area for size constancy remains undetermined to date. The results of work in monkeys suggest that size perception is mainly dependent on the occipito-temporal stream of visual information $[19,28]$.

We present here the case of a 35-year-old patient with recurrent hemimicropsia in the left hemifield resulting from a cavernous angioma in the right occipital lobe. After neurosurgical removal of the angioma, the misperception no longer occurred. Exact localization data were gathered by magnetic resonance imaging and computer-guided reconstruction techniques and were related to the clinical and behavioural findings. Functional MRI (fMRI) was employed to characterize the functional specificity of adjacent cortical areas.

\section{Clinical data}

The patient was a 35-year-old right-handed male with no history of neurological disorders in his past. As the first clinical symptom, he reported an incident where he found himself lying on the floor of his home after falling down. Subsequently he suffered from severe occipital headache, but showed no focal neurological deficits. A cranial computed tomography (CCT) in hospital showed a hyperdense lesion in his right occipital lobe, suggestive of a hemangioma or cavernoma. Since his headache disappeared within a few days, the patient did not consent to further diagnostic measures. Six weeks later, a second episode of acute headache occurred. This time the patient noticed a change in visual perception, by experiencing transient episodes of dysmetropsia in the left hemifield; he saw objects on his left smaller, shrunk and compressed and apparently farther away, though not distorted in shape. Due to the hemimicropsia, he had difficulties in perceiving proportions, symmetry, and depth, and, as a consequence, he collided with the left side of the garage when parking his car. Interestingly, objects extending into both hemifields appeared to be spatially disparate, with the left half farther away. He had no difficulties in recognizing objects or persons and did not fail to comprehend the meaning of complex pictures, as has been described in association with micropsia [15]. Further episodes lasted from $30 \mathrm{~min}$ to $2 \mathrm{~h}$ in the following days, sometimes (but not always) accompanied by headache.

In a conventional MRI investigation, the diagnosis cavernous angioma was suggested by a peripheral low signal ring (hemoglobin), $18 \mathrm{~mm}$ in diameter, and a heterogeneous central signal (vascular malformation) on T1-weighted images. The hemorrhage surrounding the cavernoma was accentuated at its superior and posterior parts, reaching well into the gray matter. It was diagnosed as a cavernous hemangioma with signs of a chronic bleeding (for details, see below).

The frequency of micropsia episodes spontaneously decreased to once or twice a week in the following months, during which the patient did not seek out further medical treatment. Four months after the first occurrence, the frequency and duration of the micropsia episodes increased anew and the patient was admitted to our hospital. Clinical-neurological examination revealed no objective deficit. A follow-up MRI showed constant findings with respect to the cavernoma. Both MR angiography and cerebral catheter angiography were normal, in line with the diagnosis cavernous angioma. Electroencephalography (EEG), consisting of a standard clinical protocol with 12 scalp electrodes placed according to the International 10-20system with unipolar and bipolar montages, was normal and gave no hint of focal epileptic seizures. In 
ophthalmologic examination, peripheral retinal diseases were excluded and the visual field was normal on Goldmann perimetry. Furthermore, visually evoked potentials showed normal P100 waves.

During the micropsia episodes the patient was unable to integrate familiar objects spatially which led to dizziness. As a consequence, psychophysical evaluations of size perception [3] during the episodes was not possible. Therefore, we resorted to retrospective reports between episodes. Interestingly, tactile information about objects that appeared to be farther away could not correct the visual impression. The smaller percept of objects on the left was dependent on the patient's visual axis and independent of his corporal axis. In the asymptomatic intervals we applied a number of visual tests used in the clinical routine, like object naming, Ishihara color test, ability to recognize known objects or persons and to comprehend the meaning of complex pictures (in the Wechsler test, [31]). All of them revealed no deficit.

Following treatment with the anti-convulsant carbamazapine, no further visual symptoms occurred. Because of the disappearance of the hemimicropsia, no further neuropsychological testings of this symptom (e.g., comparison of different-sized same objects; see [3]) could be performed. The cavernous hemangioma was neurosurgically removed without intra- and postoperative complications, and the diagnosis was histologically confirmed. As of date, six months after the operation, no visual symptoms occurred.

\section{Methods}

\subsection{Localization data}

All MRI data were acquired on a $1.5 \mathrm{~T}$ scanner (Vision, Siemens). In addition to the clinical MRI investigations (described above) we performed a 3-D reconstruction of the patient's brain and a functional MRI (fMRI) investigation. For the reconstruction, we acquired an anatomical 3-D MR data set of the patient's brain. The MR data set consisted of 150 T1weighted sagittal slices, the sequence was FLASH (TR $40 \mathrm{~ms}$, TE $6 \mathrm{~ms}$, flip angle $40^{\circ}$, FOV $256 \times 256 \mathrm{~mm}^{2}$, voxel $1 \times 1 \times 1 \mathrm{~mm}^{3}$ ). Imaging was performed using a transmit/receive full-head standard headcoil. The data set was transformed to an atlas which has been described elsewhere [33]. In short, the atlas is based upon the Visible Human (VH) data set [25] consisting of multiple $1 \mathrm{~mm}$ MR slices (resolution $768 \times 576$ pixels) of a human body in a unified coordinate system (for the brain, we used the first 165 transversal slices). The atlas was normalized with respect to the Talairach grid (see below). The segmentation capabilities were decisively improved in a further analysis which relied on non-automatic labelling of anatomic brain structures and further extrapolation. The data set was triangulated (after creating isotropic voxels) in order to reveal as many structural details as possible. Afterwards the surfaces were smoothed by a reduction of the number of triangles using both the $3: 1$ and the Eckweg-algorithm [33]. The result was refined (including surface shading) by the software REALAX (MediaSystems). For final visualization, the 3-D brain (or its individual segmented components, respectively) can be dynamically inspected in the virtual reality of REALAX.

Prior to warping the MR volume data to the atlas, they were transformed to the coordinate system of the standardized stereotaxic space by Talairach and Tournoux [26]. The reference points ac (anterior commissure) and pc (posterior commissure) were manually marked and the three reference lines ac-pc, VCA (vertical transversing ac) and midline (interhemispheric sagittal plane) were defined. Slices of the 3-D MR data set, after transformation to the Talairach stereotactic grid, are shown in Fig. 1 together with the coordinates of Talairach space. In the grid system the anatomical position of the cavernoma could be specified with regard to its exact 3-D localization and spatial relation to other structures. The cavernoma is located in the lateral part of Brodmann's area (BA) 19 of the right occipital lobe, bordering BA 37. The distances of the cavernoma center to ac were $37.5 \mathrm{~mm}, 68.0 \mathrm{~mm}$, and $1.0 \mathrm{~mm}$ in the $x$-, $y$-, and $z$-axis, respectively. The cavernoma had a volume corresponding to $692 \mathrm{~mm}^{3}$.

fMRI was performed to establish physiological landmarks with respect to visual stimulation. Our reason for testing motion perception in the fMRI was motivated by the location of the lesion. Only when we discovered the close vicinity between V5 and the lesion did we go on to test the patient's motion perception psychophysically. The perceptual function of morphologically intact areas can be tested with functional MRI (e.g., correlates of motion perception and V5 activation), whereas function of a lesioned area cannot directly be studied.

Imaging was performed with the help of a gradient system having $25 \mathrm{mT} / \mathrm{m}$ amplitude and $0.3 \mathrm{~ms}$ risetime. The subject was positioned with his head in an RF receive-transmit full headcoil. Head motion was minimized with a vacuum cap, secured within the headcoil. Local variations in blood oxygenation level dependent (BOLD) response [18] were measured using susceptibility-based fMRI imaging, applying gradientrecalled EPI sequences. Seven parallel 4-mm-thick planes, positioned in posterior cortex, were imaged every $3 \mathrm{~s}$ using a T2*-weighted sequence (TR $3000 \mathrm{~ms}$, TE $84 \mathrm{~ms}$, flip angle $90^{\circ}$, FOV $256 \times 256 \mathrm{~mm}^{2}$, matrix $128^{2}$ voxels). The positions of the planes were between axial and coronal and were chosen with the aid of a 


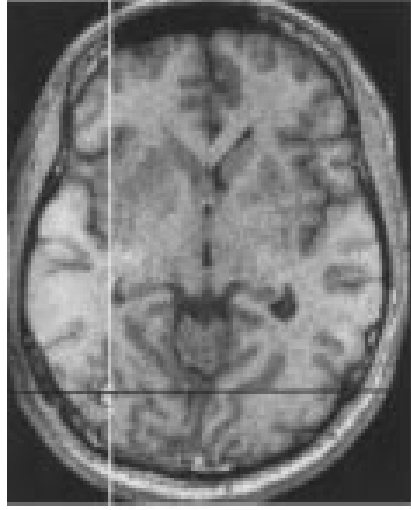

A

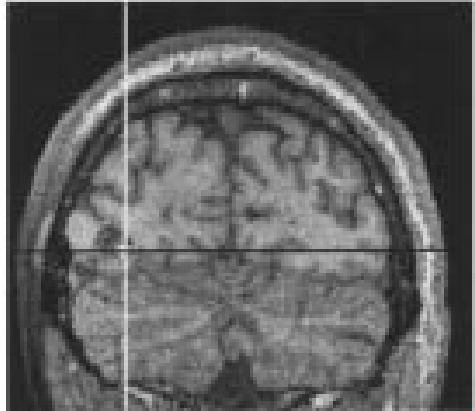

B

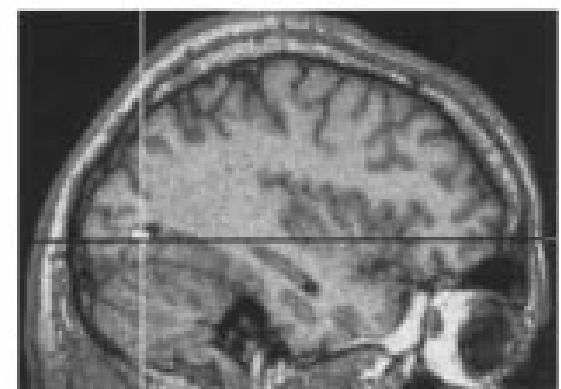

$\mathrm{C}$

Fig. 1. Slices of the 3-D MR data set, after transformation to the Talairach stereotactic grid system, in axial, coronal, and sagittal view (A, B, and $\mathrm{C}$, respectively). The center of the cavernoma is marked by the black/white crosshair, which gives the coordinates of Talairach space $(x=37.5, y=68.0$, and $z=1.0)$.

mid-sagittal T1-weighted scout image so as to include the entire occipital lobe together with posterior portions of the parietal and temporal cortex.

In the experimental fMRI session, two runs were performed, each lasting $162 \mathrm{~s}$. During this time the 7 slice volume was imaged repeatedly ( 54 volume acquisitions, $3 \mathrm{~s}$ each). The run was divided into 6 epochs of $27 \mathrm{~s}$ duration each. The three epochs containing a continuous visual stimulus (see below) were interleaved with three epochs in which the screen was unpatterned, but had the same mean luminance as the stimulus. The interleaving of 'on' and 'off' epochs enabled the activity elicited by the stimuli to be compared with the baseline activity level for each voxel in the 7-slice volume. In the first run the visual stimulus consisted of a flickering high contrast checkerboard, in the second run of a dynamic pattern with coherent motion, as described below and in a previous publication [8].

\subsubsection{Flickering checkerboard}

A random checkerboard display $(50 \%$ dark checks, $50 \%$ light checks) was presented that dynamically flickered (dark check changed to light and vice versa) at $6 \mathrm{~Hz}$. The check size was $0.5^{\circ}$ and the luminance contrast was $95 \%$. The patient was instructed to view a central fixation point during stimulus presentation in order to minimize eye movements. This stimulus has proven to yield a strong BOLD response in striate and extrastriate visual cortex. The stimulus does not provide any information about retinotopically defined area borders [22,23]. Since the patient showed no visual field defects, we suspect that the cortical tissue affected by the lesion is beyond retinotopically organized visual cortex.

\subsubsection{Random dot motion}

Sequences of random dots were presented in the expansion-contraction direction. The directional coherence level was $90 \%$. Randomly interspersed among the dots with the coherent direction were dots with a random direction (total number of dots $=100$; dot diameter $=20 \mathrm{~min}$ ). Each dot remained on the screen for no longer than $3 \mathrm{~s}$, at which time it disappeared and was replaced elsewhere by a new dot. Periods of stimulation were interleaved with rest periods, during which the subject viewed an homogenous screen of the same mean luminance. Subjects were instructed to fixate a clearly visible central stationary spot to suppress undesired eye movements during both rest and stimulation. Eye position and eye movements were not monitored during the measurements [7]. The former (checkerboard) stimulus was used to activate primarily striate and extrastriate cortex, while the latter (dot motion) stimulus was used to activate in addition the V5 region (visual motion area).

\subsection{Post-processing of fMRI data}

\subsubsection{Motion correction}

The EPI images were first corrected for head motion using the 2D motion correction algorithm IMREG, which is part of the AFNI package [4]. This algorithm realigns each image in the time series to the average position of that series. Since it performs this correction on each slice separately, it can only correct for displacements or rotations in the 2-D-plane. Visual inspection of the time series indicated that most of the residual head motion could be corrected for.

\subsubsection{BOLD response}

We analysed the data with the package BrainTools 
developed by Krish Singh. The methods have been described in detail in an earlier publication [23]. Briefly, cross correlation was performed voxelwise with the time series and a phase-shifted (6 s) smoothed $(\mathrm{SD}=3 \mathrm{~s})$ stimulus boxcar (the on-off stimulation time series). Voxels are highlighted in the functional overlays that have a correlation surpassing $Z=2.2$ $(P<0.05)$.

\subsection{Psychophysical test of coherent motion perception}

The psychophysical test of coherent motion perception consisted of a two-alternative forced-choice task, in which two fields of random dots were presented left and right of a central fixation point. One of the fields contains dots with a random direction, the other field contained a mixture of dots both with random direction and with coherent direction. The level of motion coherence was varied along four levels from 33 to $100 \%$. Psychometric functions were determined after 120 trials (30 trials for each of four coherence levels). The subjects' task was to identify the direction of the coherent motion (left or right).

\section{Results}

The results obtained from the fMRI study are shown in Fig. 2 in terms of the functional overlays. The magnitude of the BOLD effect activation level by visual stimulation is indexed by a color code. The code highlights a voxel if its time course was correlated 0.5 or better with that of a phase retarded, smooth version of the stimulus time course. A strong response is evident in the primary visual cortices (highlighted in green/yellow) with either of the two stimuli. The most lateral extentions of the activations correspond to BA 19 (estimated with reference to Talairach coordinates). In the coherent motion experiment there were, in addition, BOLD effects in a more lateral and anterior location, corresponding to the V5 area, in both hemispheres. Noticeably, when comparing the activation of V5 across the two hemispheres in the moving dots paradigm, there was a considerable difference with less activation on the side of the cavernoma. Comparing the relative strength of activation in areas representing V5 in the left and the right hemisphere across both stimulus conditions, we found $146.5 \%$ and $150.6 \%$ response in the right and the left hemisphere, respectively, in the coherently moving dots condition as compared to the flickering checkerboard condition $(100 \%)$. In a comparison across the left and right hemisphere, relative activation was lower in the right hemisphere (with the cavernoma) in both the flickering checkerboard condition $(79 \%)$ and the coherently

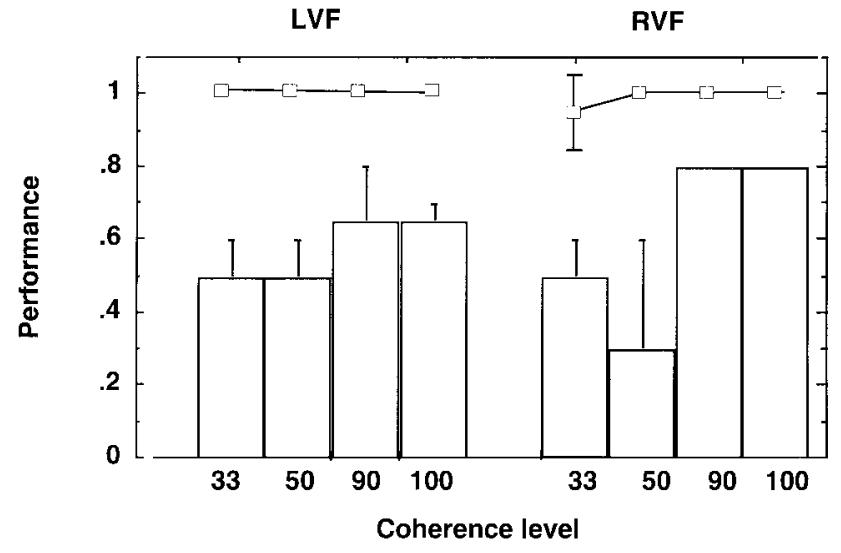

Fig. 3. Performance (portion correct) of the patient (columns) and the control subject (squares) for the four levels of direction coherence $(33-100 \%)$ in the psychophysical test of coherent motion perception. The results are shown for the left (LVF) and right (RVF) visual fields separately. Error bars show \pm 1 SE (in cases without error bars $\mathrm{SE}=0)$.

moving dots conditions (76\%) as compared to the left hemisphere $(100 \%)$.

Inter-hemispheric differences in the BOLD effect upon sensory or motor stimulation are not uncommon. We therefore asked whether the weaker BOLD effect on the right side would have some functional correlate in visual motion perception. As a consequence, we performed additional psychophysical measurements using the coherent dot motion paradigm (see above).

\subsection{Psychophysical performance on the motion coherence test}

The results of the psychophysical test of motion coherence are shown in Fig. 3. They indicate that the patient had an elevated threshold for the perception of coherent motion as compared to an age- and education-matched normal control subject $(F(1,38)=19.6$; $P<0.0001)$. Although there was a tendency for this effect to be more pronounced in the left (affected) hemifield as compared to the right hemifield, this difference was statistically not significant. We assume that this test revealed a very subtle, subclinical symptom, because the patient reported, upon request, that he never noticed disturbances of visual motion perception. Noticeably, circumscribed lesions in the temporooccipito-parietal border zone have previously shown to lead to a threshold elevation for motion perception in both visual hemifields with some preponderance of the contralateral field [9].

\section{Discussion}

This is, to our knowledge, the first report on isolated 
intermittent micropsia resulting from a small, circumscribed cortical lesion, which could be exactly localized in terms of both topological and functional anatomy. This location is in accordance with experimental findings in animals and with previous reports in the literature, as we will discuss in the following. Furthermore, we will argue that dysmetropsia reflects a disturbance of the visual size constancy mechanism, evoked by a local irritation of nervous tissue that surrounds the lesion. Probably, the symptom of intermittent micropsia experienced by our patient was caused by epileptic activity because micropsia is a rather common symptom in occipito-temporal epilepsy, whereas lesions (e.g., infarctions) are rarely accompanied by this symptom (see Introduction). The intermittent occurrence of the symptom and the immediate and enduring effect of the anticonvulsant carbamazepine further suggest an epileptic cause.

Imaging revealed the cavernous angioma as being localized in the lateral border region of BA 19. The peristriate area BA 19 is known to surround the extrastriate area BA 18, with the (primary visual) striate area BA 17 medial to them. As shown by Zeki et al. [34] in monkeys, it receives complex projections from the latter two areas. BA 19 is considered to serve an integrative visual function, by elaborating the crude sensory information received by the striate cortex as well as processing of multiple other sensory modalities [16]. After first investigations by Gross et al. [10], lesion experiments in rhesus monkeys by Ungerleider et al. [28] suggest that both prestriate cortex and inferotemporal areas are involved in the perception and discrimination underlying size constancy. From this study it appears that occipitotemporal visual pathways, nowadays often referred to as 'ventral visual stream' and considered to subserve mainly object recognition, also mediates perception of object size in relation to distance [5]. In fact, monkeys show severe deficits in size discrimination tasks following ablation of V4 [19]; note that V4 is considered by some authors as the ventral anterior part of BA 19 in human. On the one hand, visuospatial processing appears to involve mainly the 'dorsal stream' which continues into parietal cortex, with the areas of motion processing residing at the junction of the ventral and dorsal streams. We hold that the space constancy mechanism integrates information from both the ventral and the dorsal stream. On the other hand, space constancy is certainly related to our perception of objects or living beings (their perceived spatial dimension). The mechanism codes where in space we perceive an object, distant or close. This double association is not unique; the mechanism of 'form from motion' would be another example of combined information from both pathways.

In monkeys the medial temporal (MT) area is known to process visual motion information, a possible homol- ogue in humans is the visual motion area V5 [17,29,35] located in the ventral occipito-temporal cortex (4-8 $\mathrm{mm}$ below the ac-pc plane). PET and fMRI studies in humans indeed found significant activation upon visual motion stimulation in BA 37, bordering lateral BA 19, and in the ventral aspect of BA $39[23,27,30]$, depending on the directional coherence of random dot stimuli [7]. In agreement with this concept, patients with postsurgical brain lesions in this cortical region show an impaired motion perception [9].

How do these and other experimental findings relate to the clinical symptomatology of central dysmetropsia? First, we may consider that a dysmetropsia that is restricted to one hemifield, as in our case, is likely to be generated at early processing stages in the ventral visual stream, close to or in the peristriate cortex, because the receptive fields of neurons in the peristriate cortex are restricted mainly to the contralateral visual hemifield. By the same token, it is possible to speculate that whole-field dysmetropsia is more likely to arise in temporal structures, where receptive fields are generally comprising large parts of the visual field. However, these assumptions have to await future localization studies in humans. The presumed irritation of the lateral BA 19 in our patient apparently did not spread more medially to BA 18 and BA 17; at least, we had no indication for visual field defects, as one would expect from BA 17 lesions. Hemimacropsia without hemianopsia has been observed by Brégeat et al. in a patient with a tumor of the occipital lobe [2]. Anecdotal reports on impaired distance perception following occipital lesions which spared BA 17 go back to 1940 [13]. On the other hand, dysmetropsia may well be combined with (at least partial) homonymous loss of the visual field [3], because BA 17 often is affected with extended lesions in this region. Generally, dysmetropsia in earlier reports mostly was found in association with occipital lesions, due to ischemia [3], neoplasm [2], or hemorrhage [6]), but it remained for Cohen et al. [3] to localize the lesion in BA 18 and BA 19 in two patients with micropsia due to cerebral infarctions, using imaging techniques (CT, MR) and postmortem examination. However, in our study imaging localization was more accurate. In the patient studied here, the lesion was restricted to BA 19. Furthermore, we found the cavernoma in our patient to border V5. However, we consider it unlikely that micropsia arose from V5 affection, since this area has been shown to be involved mainly in visual motion processing. The patient had no complaint about abnormal motion perception, but we psychophysically revealed a minor motion perception deficit. As shown by our fMRI data, V5 is located anteriorly and laterally to the site of the lesion. Our findings thus suggest that a region neighbouring V5 must be involved in size constancy. 
Noticeably, epileptic activity was not found in the EEG recordings we performed. We hold, however, that a focal epileptic activity several millimeters in the brain does not necessarily show up in the scalp recordings. An invasive recording would have been much more promising in detecting the epileptic activity. However, since a moderate dose of carbamazepine successfully suppressed the symptom, we saw no legitimation for an invasive or semi-invasive approach.

Finally, we address the question: Why is dysmetropsia a rare complaint of patients with lesions in the occipito-temporal cortex? We assume that neural processing of the visual stimulus is not disrupted in patients with dysmetropsia, but disturbed in a specific way. We hold that, in our patient, mild bleeding of the cavernoma caused irritation of surrounding intact nervous tissue, thereby modifying the perceptual mechanism of visual size constancy.

\section{References}

[1] Bender MB, Savitsky N. Micropsia and teleopsia limited to the temporal fields of vision. Archives of Ophthalmology 1943;29:904-8.

[2] Brégeat P, Klein M, Thiébaut F, Bouniol T. Hemi-macropsie homonyme droite et tumeur occipitale gauche. Reviews in OtoNeuro-Ophthalmology 1947;19:238-40.

[3] Cohen L, Gray F, Meyrignac C, Dehaene S, Degos JD. Selective deficit of size perception, two cases of hemimicropsia. Journal of Neurology, Neurosurgery and Psychiatry 1994;57:73-8.

[4] Cox RW: AFNI. Software for analysis and visualization of functional magnetic neuroimages. Computers and Biomedical Research 1996; 29:162-173.

[5] Damasio AR. Disorders of complex visual processing. In: Mesulam MM, editor. Principles of Behavioural Neurology. Philadelphia: FA Davis, 1985.

[6] Ebata S, Ogawa M, Tanaka Y, Mizuno Y, Yoshida M. Apparent reduction in the size of one side of the face associated with a small retrosplenial hemorrhage. Journal of Neurology, Neurosurgery and Psychiatry 1991;54:68-70.

[7] Freitag P, Greenlee MW, Lacina T, Scheffler K, Radü EW. Effect of eye movements on the magnitude of fMRI responses in extrastriate cortex during visual motion perception. Experimental Brain Research 1998;119:409-14.

[8] Greenlee MW, Smith AT. Detection and discrimination of firstand second-order motion in patients with unilateral brain damage. Journal of Neuroscience 1997;17(2):804-18.

[9] Greenlee MW, Lang H-J, Mergner T, Seeger W. Visual shortterm memory of stimulus velocity in patients with unilateral posterior brain damage. Journal of Neuroscience 1995;15:2287-300.

[10] Gross CG. Visual functions of inferotemporal cortex. In: Jung R, editor. Handbook of Sensory Physiology, vol. 7/3B. Berlin, Heidelberg, New York: Springer, 1973.

[11] Hécaen H. Clinical symptomatology in right and left hemispheric lesions. In: Mountcastle VB, editor. Interhemispheric Relations and Cerebral Dominance. Baltimore: Johns Hopkins, 1962.

[12] Huber A. Eye symptoms in brain tumors. In: Huber A, editor. Eye symptoms in brain tumors, 2nd ed. Saint Louis: C V Mosby, 1971.

[13] Ingham SD. The functional relations of the primary, secondary, and tertiary visual cortical areas. Bulletin of the LA Neurological Society 1940;5:12-7.
[14] Michaeli-Cohen A, Almog Y, Loewenstein A, Stolovitch C, Gutman I, Lazar M. Presumed ocular myasthenia and micropsia, a case report. Journal of Neuro-Ophthalmology 1996;16:18-20.

[15] Miller NR. Clinical Neuro-Ophthalmology, 4th edn. Baltimore/ London: Williams and Wilkins, 1982.

[16] Mishkin M, Ungerleider LG, Macko KA. Object vision and spatial vision, two cortical pathways. Trends in Neuroscience 1983;6:414-7.

[17] Newsome WT, Paré EB. A selective impairment of motion perception following lesions of the middle temporal visual area (MT). Journal of Neuroscience 1988;8:2201-11.

[18] Ogawa S, Lee TM, Nayak AS, Glynn P. Oxygenation-sensitive contrast in magnetic resonance image of rodent brain at high magnetic fields. Magnetic Resonance in Medicine 1990;14:68-78.

[19] Schiller PH, Lee K. The role of the primate extrastriate area V4 in vision. Science 1991;251:251-3.

[20] Schneck JM. Psychogenic micropsia. Psychiatric Quarterly 1971;45:542-4.

[21] Shevell MI. Acephalgic migraines of childhood. Pediatric Neurology 1996;14:211-5.

[22] Sereno MI, Dale AM, Reppas JB, Kwong KK, Belliveau JW, Brady TJ, Rosen BR, Tootell RB. Borders of multiple visual areas in humans revealed by functional magnetic resonance imaging. Science 1995;268:889-93.

[23] Smith AT, Greenlee MW, Singh KD, Krämer FK, Hennig J. The processing of first- and second-order motion in human visual cortex assessed by fMRI. Journal of Neuroscience 1998;18(10):3816-30.

[24] Smith G, Meehan JW, Day RH. The effect of accommodation on retinal image size. Human Factors 1992;34:289-301.

[25] Spitzer V, Ackerman MJ, Schersinger AL, Whitlock D. The Visible Human Male-A technical report. Journal of the American Medical Informatics Association 1996;3:118-30.

[26] Co-Planar Atlas of the Human Brain. In: Talairach J, Tournoux P, editors. 3-Dimensional Proportional System. Stuttgart-New York: Thieme, 1988.

[27] Tootell RBH, Kwong KK, Belliveau JW. Functional analysis of human MT and related visual cortical areas using MRI. Journal of Neuroscience 1995;15:3215-30.

[28] Ungerleider LG, Ganz L, Pribram KH. Size constancy in rhesus monkeys, effects of pulvinar, prestriate, and inferotemporal lesions. Experimental Brain Research 1977;27:251-69.

[29] Van Essen DC, Newsome WT, Bixby JL. The pattern of interhemispheric connections and its relationship to extrastriate visual areas in the macaque monkey. Journal of Neuroscience 1982;2:265-83.

[30] Watson JDG, Myers R, Frackowiak RSJ, Hajnal JV, Woods RP, Mazziotta JC, Shipp S, Zeki S. Area V5 in the human brain, evidence from a combined study using PET and MRI. Cerebral Cortex 1993;3:79-84.

[31] Wechsler D. WMS-R: Wechsler Memory Scale-Revised Manual. San Antonio, Texas. Harcourt Brace Jovanovich: The Psychological Corporation, 1987.

[32] Wieser HG. Zur Frage der lokalisatorischen Bedeutung epileptischer Halluzinationen. In: Karbowski $\mathrm{K}$, editor. Halluzinationen bei Epilepsien und ihre Differentialdiagnose. Bern-Stuttgart-Wien: Hans Huber, 1982. p. 67-92.

[33] Wolter T, Otte M, Jaeger D, Mergner T. Ein hochaufgeloester digitaler Gehirnatlas. In: Arnolds B, Mueller H, Saupe D, Tolxdorff $\mathrm{T}$, editors. Digitale Bildverarbeitung in der Medizin. Freiburg University Press, 1997.

[34] Zeki SM. The cortical projections of foveal striate cortex in the rhesus monkey. Journal of Physiology 1978;277:227-44.

[35] Zeki SM. Functional specialisation in the visual cortex of the rhesus monkey. Nature 1978;274:423-8. 